\title{
Repulsive Casimir Effect with Chern Insulators
}

\author{
Pablo Rodriguez-Lopez ${ }^{1}$ and Adolfo G. Grushin ${ }^{2,3}$ \\ ${ }^{1}$ Department of Physics and GISC, Loughborough University, Loughborough LE11 3TU, United Kingdom \\ ${ }^{2}$ Max-Planck-Institut für Physik komplexer Systeme, Nöthnitzer Strasse 38, 01187 Dresden, Germany \\ ${ }^{3}$ Instituto de Ciencia de Materiales de Madrid, CSIC, Cantoblanco, E-28049 Madrid, Spain \\ (Received 9 October 2013; revised manuscript received 5 November 2013; published 7 February 2014)
}

\begin{abstract}
We theoretically predict that the Casimir force in vacuum between two Chern insulator plates can be repulsive (attractive) at long distances whenever the sign of the Chern numbers characterizing the two plates are opposite (equal). A unique feature of this system is that the sign of the force can be tuned simply by turning over one of the plates or alternatively by electrostatic doping. We calculate and take into account the full optical response of the plates and argue that such repulsion is a general phenomena for these systems as it relies on the quantized zero frequency Hall conductivity. We show that achieving repulsion is possible with thin films of $\mathrm{Cr}$-doped $(\mathrm{Bi}, \mathrm{Sb})_{2} \mathrm{Te}_{3}$, that were recently discovered to be Chern insulators with quantized Hall conductivity.
\end{abstract}

More than half a century after its theoretical prediction, the Casimir effect [1] still stands among the most intriguing quantum phenomena. The relatively recent quantitative experimental access to the physics of this effect [2], the force experienced by objects due to quantum vacuum fluctuations, has revealed that it is still far from being completely understood. Despite the development of useful calculating tools in terms of the scattering formalism [3,4], the possibility of achieving repulsion in vacuum between two material plates is still so far unreachable experimentally. Two dielectrics can repel when immersed in a medium with very specific optical properties [5,6] and no mirror-symmetric situation can give rise to repulsion $[7,8]$. These restrictions turn the search for repulsion in vacuum into a difficult challenge that can potentially solve stiction issues [2,9]. Earlier proposals include magnetic materials [10], metamaterials [11,12], engineered geometries [13], and quantum Hall effect (QHE) systems [14], where the latter was subsequently generalized to a QHE system made out of doped graphene sheets [15]. In $[16,17]$, the concept of a topological Casimir effect was explored using three-dimensional topological insulators (TI) $[18,19]$, which owing to their topological electromagnetic (EM) response, opened the way to a tunable repulsion. In these works, the finite frequency part of the topological response [20] arising from the EM response encoded in the $\theta$ term [21,22] was assumed to be the quantized zero frequency response for all frequencies, which is only valid for certain distance scales depending on material parameters.

In this Letter, we propose to achieve and manipulate repulsion by exploring the Casimir force arising due to the topological nature of Chern insulators (CI). These general class of two dimensional materials have a quantized Hall conductivity in the absence of external magnetic field due to the nontrivial topological structure of the Bloch bands $[18,19,23]$. The Chern number $C \in \mathbb{Z}$ is the topological attribute of each band that, if finite, indicates a quantized contribution to the Hall conductivity at zero frequency $\sigma_{x y}(0)=C e^{2} / h$. Motivated by the recent discovery of this phase in Cr-doped $\mathrm{TI}(\mathrm{Bi}, \mathrm{Sb})_{2} \mathrm{Te}_{3}$ [24], in this Letter, we show that the use of this class of materials is a feasible possibility to overcome the strict theoretical bounds to realize Casimir repulsion in realistic systems. We also show that these systems are unique in terms of controlling and reversing in a simple way the repulsive force.

We will first derive the long and short distance limits for the Casimir force of a generic CI lattice model. Under general assumptions, we show that whenever the sign of the Chern number characterizing the two CI plates is opposite (i.e., unequal signs of the zero frequency Hall conductivity) the system realizes Casimir repulsion at long distances. We further support this result by obtaining numerically the Casimir energy density (CED) for Casimir plates described by generic $\mathrm{CI}$ lattice models with different Chern numbers, that can be tuned by controlling the TI thin film thickness [25-27] or by achieving topological layered [28] or multiorbital models [29]. Starting from a lattice model enables us to take into account the complete frequency dependence of the electronic response functions [in this case, the conductivity tensor $\sigma_{i j}(\omega)$ ] previously overlooked [15-17], and a key issue to ascertain any realistic Casimir force prediction [6]. The length scale from which repulsion is achieved is inversely proportional to the products of the single particle gap and the Chern number of both plates. Finally, we show that the scaling law governing the CED strongly depends on whether the Chern number of the plates is finite or zero. Based on these results, we discuss the possibility of achieving repulsion in the recently discovered CI in Cr-doped $(\mathrm{Bi}, \mathrm{Sb})_{2} \mathrm{Te}_{3}[24,26,27]$ and related systems.

The CED $E(d)$ between two plates separated by a distance $d$ is given by $[2,3,5,30]$ 


$$
\frac{E(d)}{\hbar}=\int_{0}^{\infty} \frac{d \xi}{2 \pi} \int \frac{d^{2} \mathbf{k}_{\|}}{(2 \pi)^{2}} \log \operatorname{det}\left[1-\mathbf{R}_{1} \cdot \mathbf{R}_{2} e^{-2 k_{z} d}\right] .
$$

Here, $k_{z}=\sqrt{\boldsymbol{k}_{\|}^{2}+\xi^{2} / c^{2}}$ is the wave vector perpendicular to the plates, $\boldsymbol{k}_{\|}$is the momentum parallel to the plates, and $\xi$ is the imaginary frequency $\omega=i \xi$. The $2 \times$ 2 reflection matrices $\mathbf{R}_{1,2}$ contain the Fresnel coefficients

$$
\mathbf{R}=\left[\begin{array}{ll}
R_{s s}\left(i \xi, \mathbf{k}_{\|}\right) & R_{s p}\left(i \xi, \mathbf{k}_{\|}\right) \\
R_{p s}\left(i \xi, \mathbf{k}_{\|}\right) & R_{p p}\left(i \xi, \mathbf{k}_{\|}\right)
\end{array}\right]
$$

The matrix elements $R_{i, j} \quad i, j=s, p$ describe parallel (perpendicular) polarization of the electric field with respect to the plane of incidence. The Casimir force per unit area on the plates is obtained by differentiating expression Eq. (1) $F=-\partial_{d} E(d)$. A positive (negative) force, corresponds to repulsion (attraction).

The $R_{i, j}$ components for a generic system can be computed by solving Maxwell's equations in the presence of the plate imposing boundary conditions (see [31]). For a two dimensional system described by $\sigma_{i j}(\omega)$, we find [15]

$$
\begin{aligned}
& R_{s s}=-\frac{2 \pi}{\Delta}\left[\frac{\sigma_{x x}}{\lambda}+2 \pi\left(\sigma_{x x}^{2}+\sigma_{x y}^{2}\right)\right], \\
& R_{s p}=R_{p s}=\frac{2 \pi}{\Delta} \sigma_{x y}, \\
& R_{p p}=\frac{2 \pi}{\Delta}\left[\lambda \sigma_{x x}+2 \pi\left(\sigma_{x x}^{2}+\sigma_{x y}^{2}\right)\right],
\end{aligned}
$$

with $\Delta=1+2 \pi \sigma_{x x}[(1 / \lambda)+\lambda]+4 \pi^{2}\left(\sigma_{x x}^{2}+\sigma_{x y}^{2}\right)$ and $\lambda=$ $k_{z} / \omega$ in units where $c=1$. In our convention, these are consistent with results for 3D TI $[16,32,33]$ and are related to those of Ref. [15] by a basis transformation. To evaluate Eq. (1), we employ the generic model used in Ref. [34] for the CI plates. This family of two-band models captures the characteristic low energy features of any CI, (i) a quantized
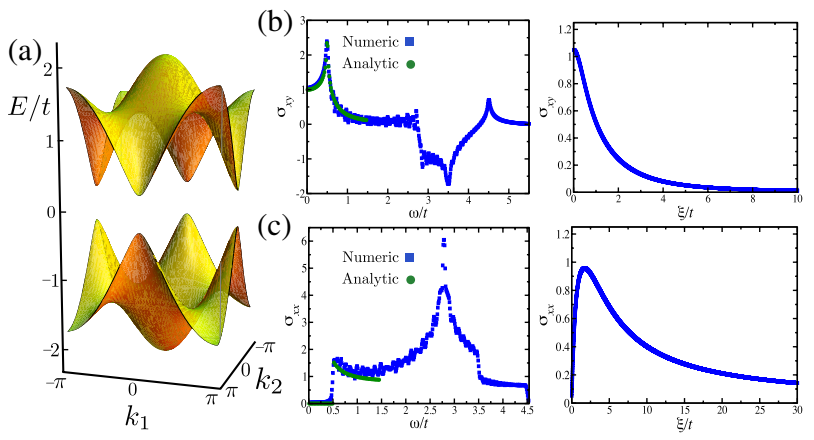

dc Hall conductivity $\sigma_{x y}(0)=C e^{2} / h$, where $C$ is a quantized topological integer, the Chern number of the lower band $[18,19,23]$ and (ii) the insulating behavior $\sigma_{x x}(0)=0$. The model also naturally takes into account the effect of a finite bandwidth since it is defined from a two band tight-binding model for fermions on a two-dimensional square lattice, with Hamiltonian

$$
\begin{aligned}
H_{0}= & \sum_{k \in \mathrm{BZ}} c_{k}^{\dagger} d_{\boldsymbol{k}} \cdot \boldsymbol{\sigma} c_{\boldsymbol{k}}, \\
d_{k ; 1}+\mathrm{i} d_{k ; 2}= & t\left(\sin k_{1}+\mathrm{i} \sin k_{2}\right), \\
d_{k ; 3}= & h_{1} \cos k_{1}+h_{2} \cos k_{2}+h_{3} \\
& +2 h_{4} \cos k_{1} \cos k_{2},
\end{aligned}
$$

where $c_{\boldsymbol{k}}^{\dagger} \equiv\left(c_{\boldsymbol{k}, \uparrow}^{\dagger}, c_{\boldsymbol{k}, \downarrow}^{\dagger}\right)$ and $c_{\boldsymbol{k}, s}^{\dagger}$ creates a fermion at momentum $\boldsymbol{k}$ in the Brillouin zone (BZ) with $s=\uparrow, \downarrow$ being the spin or sublattice degree of freedom and $\sigma=$ $\left(\sigma_{1}, \sigma_{2}, \sigma_{3}\right)$ are the Pauli matrices. The hopping parameters $t$ and $h_{\mu}, \mu=1, \ldots, 4$, are real and can be determined by optical spectroscopy. This model has, at low energies, four gapped Dirac fermions that contribute $\pm 1 / 2$ to the total Chern number (see [31]). In that way, tuning $h_{\mu}$ leads to different CI with different sizes of the single particle gap and Chern numbers. Thus, the Chern number of each CI plate can be chosen to take the values $C=\{0, \pm 1, \pm 2\}$ for generic single particle gap sizes $m$. Using the Kubo formula, we have calculated $\sigma_{i j}(\omega)$ for the model Eq. (4) at all frequencies and then used the Kramers-Kronig relations to find $\sigma_{i j}(\omega=i \xi)$ exactly. We have also checked that the latter is equivalent to evaluating the Kubo formula at imaginary frequencies. Representative results together with a typical band structure are shown in Figs. 1(a)-1(c) (see [31]). The complete tight-binding calculation of the optical signatures of a lattice model of a CI is the first result of this Letter and will be used to numerically evaluate Eq. (1).

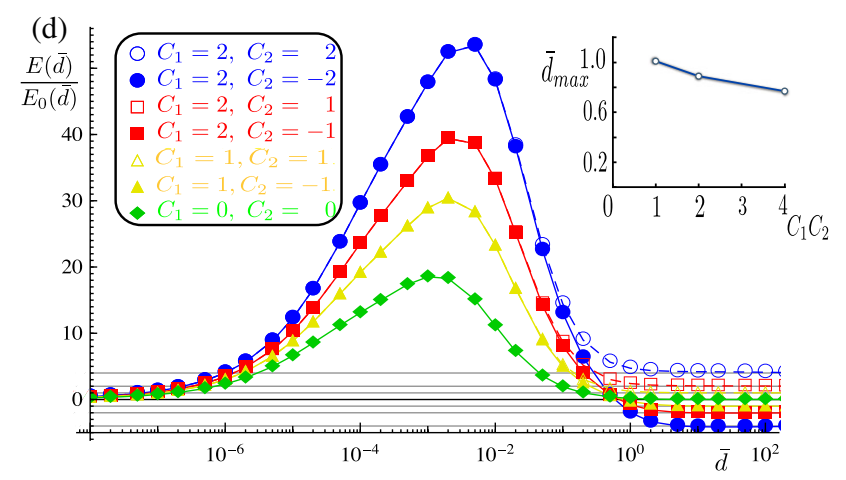

FIG. 1 (color online). (a) Band structure, and real part of (b) $\sigma_{x y}(\omega)$ and (c) $\sigma_{x x}(\xi)$ for Eq. (4) calculated for $h_{\mu}=(1,1,0.25,0) t$. The bands have $C= \pm 1$ and the conductivities are given in units of $e^{2} / h$. A comparison is shown with the analytical formulas for Dirac fermions (see [31]). (d) $E(d)$ in units of $E_{0}(d)=-\hbar c \alpha^{2} /\left(8 \pi^{2} d^{3}\right)$ as a function of $\bar{d}=d /(\hbar c / t)$. For a CI with $C=\{0, \pm 1, \pm 2\}$, $h_{\mu} / t=\{(0,0,1,0),(1,1, \pm 1,0),(0,0,0, \pm 1)\}$ were chosen, respectively, all with $m / t=1$. Inset: $d_{\max }$ of $E(d)$ as a function of $C_{1} C_{2}$. 
Before proceeding, it is possible to predict the behavior of the Casimir effect for a generic Casimir system built up of two CI with Chern numbers $C_{1,2}$ from the asymptotic properties of $\sigma_{i j}(\omega)$. At short distances (large frequencies), these materials behave as ordinary dielectrics which implies attraction $[7,8]$. For low frequencies (long distances) however, the longitudinal conductivity vanishes, since $\mathrm{CI}$ are insulators and we are left only with a quantized $\sigma_{x y}^{(i)}=C_{i} e^{2} / h$ for each plate. Introducing these into Eq. (1), we obtain (see [31])

$$
\begin{aligned}
E(d)= & -\frac{\hbar c \alpha^{2}}{8 \pi^{2} d^{3}} C_{1} C_{2}-\frac{9 \hbar c \alpha^{2}}{10 d^{5}} b_{1} b_{2}, \\
& -\frac{\hbar c \alpha^{3}}{4 \pi d^{4}}\left[C_{1}^{2} b_{2}+C_{2}^{2} b_{1}-2 C_{1} C_{2}\left(b_{1}+b_{2}\right)\right],
\end{aligned}
$$

$\left(\alpha=e^{2} / \hbar c\right)$ which is valid for distances larger than the length scale set by $1 / b_{i} \sim 2 m_{i}$, the single particle gap, and for $C_{i}$ of order 1 . For two CI plates with opposite Chern numbers, this result implies repulsion at long distances. The strength of this statement is that it does not depend on the specific model of CI (in particular, the number of bands, the concrete material realization, etc.), since it only relies on the quantized Hall conductivity and insulator properties, which are present given that the material is a CI. The key issue is, therefore, to understand at what distances we can expect such a repulsive behavior in real materials to exist. To answer this question, we have characterized precisely the crossover from repulsion to attraction by numerically computing the CED for the model Eq. (4). We use the dimensionless distance $\bar{d}=d /(\hbar c / t)$, where $t$ is the hopping (typically $t \sim 1 \mathrm{eV}$ and $\bar{d} \sim 1$; $d \sim 0.2 \mu \mathrm{m})$. In this calculation, we include the complete numerical $\sigma_{i j}(\omega)$. In Fig. 1(d), we present the CED as a function $\bar{d}$ between two $\mathrm{CI}$ plates characterized by Chern numbers $C_{1,2}$. To unravel the effect of changing the Chern number, we have chosen for this case the parameters $h_{\mu}$ such that both CI plates have the same single particle gap $m / t=1$. For all cases, we obtain repulsion (attraction) at long distances and attraction at short distances as long as $C_{1} C_{2}<0\left(C_{1} C_{2}>0\right)$. All curves recover the analytic result Eq. (5) at long distances. The Casimir force is strongly suppressed when $C_{i}=0$. The effect of changing the single particle gap $m$ while keeping $\left|C_{1}\right|=\left|C_{2}\right|$ constant is shown in Fig. 2(a). In this case, we observe the same crossover behavior as long as the Chern numbers have opposite signs.

In order to optimize possible experimental systems discussed below, we address the question on the dependence of the position maximum $d_{\max }$ of $E(d)$ with the different parameters. The point $d_{\max }$ results from the interplay between $\sigma_{x y}(\omega)$ and $\sigma_{x x}(\omega)$ in Eq. (3). By expanding both for $\omega / t \ll 1$, it is simple to estimate from Eq. (3) that $d_{\max } \sim 1 / \sqrt{\left|C_{1} C_{2}\right| m_{1} m_{2}}$ with a coefficient of order 1 and as long both $C_{i} \neq 0$ (see [31]). The numerical evidence for this qualitative behavior is shown in the insets of Figs. 1 (d) and 2(a). The former shows that $d_{\max }$ indeed decreases with $\left|C_{1} C_{2}\right|$. For the model Eq. (4), each Chern number, when finite, can only take the values $C_{i}=\{ \pm 1, \pm 2\}$, and so $\left|C_{1} C_{2}\right|=\{1,2,4\}$, providing very few points to guarantee a good fit for the power law behavior discussed above. It is, therefore, more useful to study the change of $d_{\max }$ against the product of the two single particle gaps $m_{1} m_{2}$ which can be tuned easily by modifying the vector $h_{\mu}$. The results are shown in the inset of Fig. 2(a). We find that the best fit to $d_{\max }=\alpha /\left(m_{1} m_{2}\right)^{\beta}$ is achieved for $\beta=$ 0.41 and $\alpha=0.96$ for $C_{1}=-C_{2}=1$, providing evidence in favor of the simple relation above. Small deviations originate from higher values of $m_{1} m_{2}$ that might not follow this simple law. We present also $d_{\max }$ as a function of the product of flatness ratios $\delta_{1} \delta_{2}$ with $\delta_{i} \equiv W_{i} / 2 m_{i}$, where $W_{i}$ is the bandwidth of the filled band.

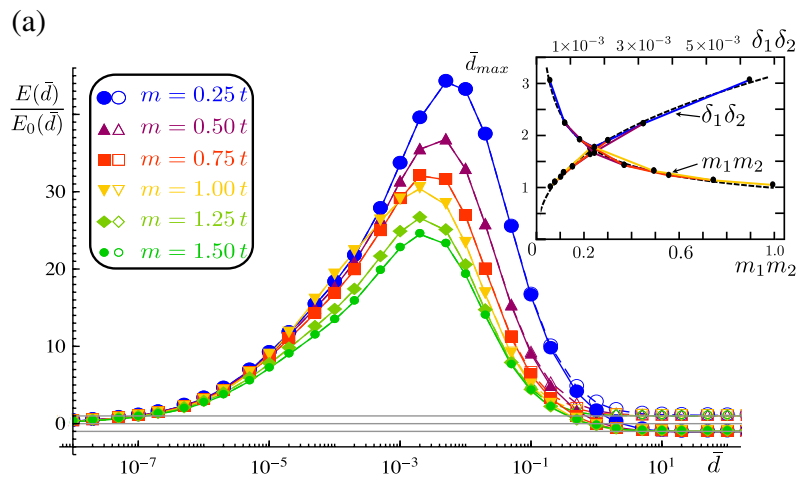

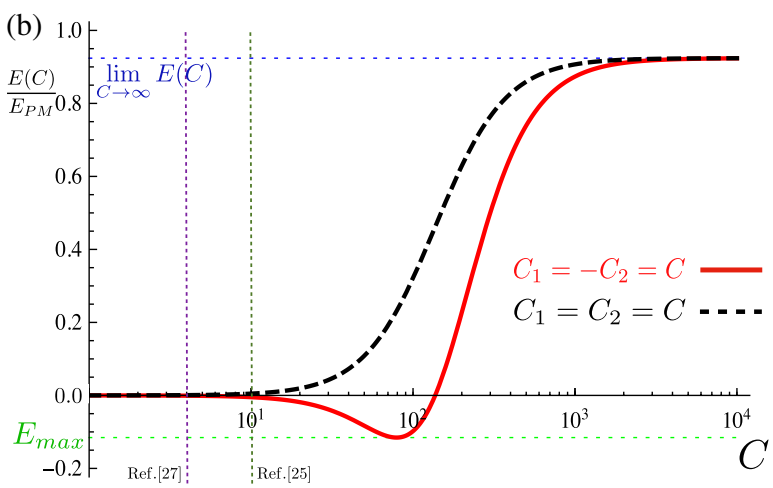

FIG. 2 (color online). (a) $E(d)$ in units of $E_{0}(d)=-\hbar c \alpha^{2} /\left(8 \pi^{2} d^{3}\right)$ as a function $\bar{d}=d /(\hbar c / t)$ for different values of the single particle gaps $m / t$. Different curves represent CI plates with $\left|C_{i}\right|=1$ and single particle gaps given by $m / t \equiv m_{1} / t=m_{2} / t=\{0.25,0.5,1.0,1.25,1.5\}$. Inset: $d_{\max }$ of $E(d)$ as a function of the gap and flatness ratio products, $m_{1} m_{2}$ and $\delta_{1} \delta_{2}$, respectively. The colors indicate values of $m_{1}$. The black-dashed line is a fit to $d_{\max } \sim 0.96 /\left(m_{1} m_{2}\right)^{0.41}$ and $d_{\max } \sim 23.51\left(\delta_{1} \delta_{2}\right)^{0.41}$. (b) Long distance limit behavior of the CED for two CI in units of the perfect metal result. Chern numbers to the left of the vertical dashed lines are achievable Chern numbers following [27] and a conservative estimate of [25]. 
In addition, the leading contribution in Eq. (5) vanishes if either or both $C_{i}=0$. From the first nonzero contribution of Eq. (1), we find that if either one (both) of the Chern numbers is (are) zero the Casimir scales analytically as $\sim 1 / d^{4}\left(\sim 1 / d^{5}\right)$. Therefore, fixing $d$ but changing from a configuration with $C_{1}=C_{2}$ to one with either or both $C_{i}=0$ will also reveal the effect of a finite Chern number. On the short distance limit, we find analytically that $E(d) \sim$ $1 / d^{5 / 2}$ and independent of $C_{i}$. For both limits, the analytical and numerical calculations agree both quantitatively and qualitatively (see [31]).

One of the most promising candidates to realize repulsion is the recently discovered CI phase in Cr-doped $(\mathrm{Bi}, \mathrm{Sb})_{2} \mathrm{Se}_{3}$ [24]. Experimentally, this material has $\sigma_{x y}(0)=e^{2} / h$ and $\sigma_{x x}(0) \sim 0$. Thus, a CI model such as Eq. (4) captures the low energy properties since the chemical potential can be tuned to lie inside the single particle gap with a gate voltage [24]. Typical experimental values for measurable Casimir pressures and distances are $\mathrm{pN} / \mathrm{m}^{2}$ and $\mu \mathrm{m}$, respectively [2]. For a CI of the type discovered in Ref. [24], the single particle gaps are of the order of $\sim 0.02 \mathrm{eV}$ [35] and Chern numbers up to $|C|=4[26,27]$ or even $|C| \gtrsim 10$ [25] could be reached in the thin film setup. Note that from Eq. (5), increasing $C$ can result in stronger forces. However, as shown in Fig. 2(b), the behavior for sufficiently high Chern numbers, beyond the validity of Eq. (5), can be different since (i) there exists an optimal Chern number $C_{\max } \simeq 1 /(\sqrt{3} \alpha)$ for which the repulsive Casimir energy is maximum reaching $\sim 10 \%$ of the value for perfect metallic plates and (ii) the force turns attractive beyond $C_{0} \simeq 1 / \alpha$.

Combining together our results, we now establish an estimate for the physical realization of the effect. For two CI plates with Chern number $C \sim 10$ [25] and single particle gap $m_{i}=50 \mathrm{meV}$, the crossover lies at a distance of $d_{\max } \sim 0.39 \mu \mathrm{m}$. At the vicinity of the maximum, the typical magnitude of the pressure is a factor $10^{-2}$ smaller that of the metal-metal Casimir pressure $\left[E_{P M}(d)=\right.$ $\left.-\hbar c \pi^{2} /\left(720 d^{3}\right)\right]$ and 1 order of magnitude bigger than that of graphene [15]. Although close to experimental limits, the resulting Casimir pressure at such a separation is still within observable bounds [36]. Alternatively, multiorbital [29] or multilayer materials $[25,28]$ with possible larger gaps can bring the force even further within measurable values.

We finish with some general remarks. Firstly, repulsion is determined by the relative chirality of the edge states of each plate, which also establish the sign of the off diagonal Fresnel coefficients. Since the Hall effect is not induced externally, turning over one of the plates (i.e., pointing its normal in the opposite direction $\hat{n}\|\hat{z} \rightarrow \hat{n}\|-\hat{z}$ ) will then change the sign of the off diagonal Fresnel coefficients. This is equivalent to reversing the sign of one $C_{i}$ and hence, can turn attraction into repulsion and viceversa. This is an exclusive and differentiating feature of CI as compared to QHE systems arising from external magnetic fields [15], and endows the CI system with a remarkably simple way of manipulating the sign of the force. Secondly, if the chemical potential crosses a band, the plates will be metallic but still have a finite Hall conductivity [37]. In this case, the force would be attractive due to the dominant Fermi surface contribution of $\sigma_{x x}(0)$ and $10^{3}$ times larger than for the insulating case. Thus, doping electrostatically either plate switches repulsion to attraction. Also, our results and Eq. (4) also can serve as a first approximation to model the surface contribution at finite frequency [20] of 3D TIs [16]. Interpreting our findings for the particular case where $C_{1,2}= \pm 1$ as a zero magnetic field analogue of the QHE system $[14,15]$ has to be understood with caution since (i) there is a sign difference in Eq. (5) that prevents the simple mapping $C_{i} \rightarrow \nu_{i}$, where $\nu_{i}$ is the filling fraction and (ii) the possibility of tuning the sign of the force by simply turning over one of the plates is exclusive to the CI system. Moreover, the refinement and complexity of Casimir experiments makes the disposal of the external magnetic field a particularly valuable feature of the proposed CI system. The repulsive behavior discussed here also applies to the fractional version of Chern insulators [38-40].

To conclude, we have shown that a CI system realizes a repulsive Casimir effect that can be tuned to attraction by simply turning over one of the plates or by electrostatic doping. Our results point towards TI thin films and other systems with higher Chern numbers [25-29] as the most promising future route to realize and control Casimir repulsion.

We thank A. Cortijo, F. de Juan, M. A. H. Vozmediano, and W.-K. Tse for discussions and Diego Dalvit and Frank Pollmann for critical reading of the manuscript. Support from FIS2011-23713, PIB2010BZ-00512 (A. G. G.) and EPSRC under EP/H049797/1 and MOSAICO (P. R.-L.) is acknowledged.

[1] H. B. G. Casimir, Proc. K. Ned. Akad. Wet. 51, 793 (1948).

[2] M. Bordag, G. Klimchitskaya, U. Mohideen, and V. Mostepanenko, Advances in the Casimir Effect (Oxford University Press, New York, 2009).

[3] S. J. Rahi, T. Emig, N. Graham, R. L. Jaffe, and M. Kardar, Phys. Rev. D 80, 085021 (2009).

[4] A. Lambrecht, P. A. M. Neto, and S. Reynaud, New J. Phys. 8, 243 (2006).

[5] I. Dzyaloshinskii, E. M. Lifshitz, and L. P. Pitaevskii, Adv. Phys. 10, 165 (1961).

[6] S. J. Rahi, M. Kardar, and T. Emig, Phys. Rev. Lett. 105, 070404 (2010).

[7] O. Kenneth and I. Klich, Phys. Rev. Lett. 97, 160401 (2006).

[8] C. P. Bachas, J. Phys. A 40, 9089 (2007).

[9] J. Munday, F. Capasso, and V.A. Parsegian, Nature (London) 457, 170 (2009).

[10] T. H. Boyer, Phys. Rev. A 9, 2078 (1974). 
[11] F. S. S. Rosa, D. A. R. Dalvit, and P. W. Milonni, Phys. Rev. Lett. 100, 183602 (2008).

[12] R. Zhao, J. Zhou, T. Koschny, E. N. Economou, and C. M. Soukoulis, Phys. Rev. Lett. 103, 103602 (2009).

[13] M. Levin, A. P. McCauley, A. W. Rodriguez, M. T. H. Reid, and S. G. Johnson, Phys. Rev. Lett. 105, 090403 (2010).

[14] M. Bordag and D. Vassilevich, Phys. Lett. A 268, 75 (2000).

[15] W.-K. Tse and A. H. MacDonald, Phys. Rev. Lett. 109, 236806 (2012).

[16] A. G. Grushin and A. Cortijo, Phys. Rev. Lett. 106, 020403 (2011).

[17] A. G. Grushin, P. Rodriguez-Lopez, and A. Cortijo, Phys. Rev. B 84, 045119 (2011).

[18] M. Z. Hasan and C. L. Kane, Rev. Mod. Phys. 82, 3045 (2010).

[19] X.-L. Qi and S.-C. Zhang, Rev. Mod. Phys. 83, 1057 (2011).

[20] A. G. Grushin and F. de Juan, Phys. Rev. B 86, 075126 (2012).

[21] X.-L. Qi, T. L. Hughes, and S.-C. Zhang, Phys. Rev. B 78, 195424 (2008).

[22] A. M. Essin, J. E. Moore, and D. Vanderbilt, Phys. Rev. Lett. 102, 146805 (2009).

[23] F. D. M. Haldane, Phys. Rev. Lett. 61, 2015 (1988).

[24] C.-Z. Chang, J. Zhang, X. Feng, J. Shen, Z. Zhang, M. Guo, K. Li, Y. Ou, P. Wei, L.-L. Wang et al., Science 340, 167 (2013).

[25] H. Jiang, Z. Qiao, H. Liu, and Q. Niu, Phys. Rev. B 85, 045445 (2012).
[26] J. Wang, B. Lian, H. Zhang, Y. Xu, and S.-C. Zhang, Phys. Rev. Lett. 111, 136801 (2013).

[27] C. Fang, M. J. Gilbert, and B. A. Bernevig, arXiv:1306.0888.

[28] M. Trescher and E. J. Bergholtz, Phys. Rev. B 86, 241111 (2012).

[29] S. Yang, Z.-C. Gu, K. Sun, and S. D. Sarma, Phys. Rev. B 86, 241112 (2012).

[30] M. T. Jaekel and S. Reynaud, J. Phys. I (France) 1, 1395 (1991).

[31] See Supplemental Material at http://link.aps.org/ supplemental/10.1103/PhysRevLett.112.056804 for further technical details.

[32] Y. N. Obukhov and F. W. Hehl, Phys. Lett. A 341, 357 (2005).

[33] M.-C. Chang and M.-F. Yang, Phys. Rev. B 80, 113304 (2009).

[34] A. G. Grushin, T. Neupert, C. Chamon, and C. Mudry, Phys. Rev. B 86, 205125 (2012).

[35] H.-Z. Lu, A. Zhao, and S.-Q. Shen, Phys. Rev. Lett. 111, 146802 (2013).

[36] F. Intravaia, S. Koev, I. W. Jung, A. A. Talin, P. S. Davids, R. S. Decca, V. A. Aksyuk, D. A. R. Dalvit, and D. Lopez, Nat. Commun. 4, 2515 (2013).

[37] F. D. M. Haldane, Phys. Rev. Lett. 93, 206602 (2004).

[38] T. Neupert, L. Santos, C. Chamon, and C. Mudry, Phys. Rev. Lett. 106, 236804 (2011).

[39] E. Tang, J.-W. Mei, and X.-G. Wen, Phys. Rev. Lett. 106, 236802 (2011)

[40] K. Sun, Z. Gu, H. Katsura, and S. D. Sarma, Phys. Rev. Lett. 106, 236803 (2011). 